\title{
¿CÓMO SOBREVIVIR A LA EXCELENCIA DEL DEPORTE DURANTE EL CONFINAMIENTO POR LA COVID-19?
}

\author{
David Moscoso-Sánchez \\ Universidad de Córdoba \\ José Carlos Jaenes Sánchez \\ David Alarcón Rubio \\ Universidad Pablo de Olavide
}

\section{RESUMEN}

En el presente artículo se analizan los resultados de una encuesta realizada durante el confinamiento de 2020 a deportistas españoles federados, de alto nivel y olímpicos. A través del cuestionario Reacciones emocionales y adaptativas en el confinamiento COVID-19 (REACOVID-19), distribuido mediante federaciones y clubes deportivos a una muestra no probabilística de bola de nieve, fueron entrevistados 2.831 deportistas. Se les consultó sobre sus condiciones de hábitat, familiares y ocio durante el confinamiento, los hábitos y comportamientos de entrenamiento, las actitudes relacionadas con la motivación y organización de sus ejercicios, así como la disponibilidad de materiales, el seguimiento de entrenadores y la información recibida por federaciones y clubes deportivos. Por último, también se les consultó acerca de su posición y el modo como les ha afectado la suspensión de los Juegos Olímpicos de Tokio 2020. Además de establecer una fotografía sobre la situación de entrenamiento de estos deportistas en dicho contexto, en el artículo se exponen las dificultades y limitaciones de realizar una encuesta de esta naturaleza en situación de estado de alarma.

Palabras clave: Deporte federado, de alto nivel y olímpico; Encuesta; Confinamiento; Crisis epidemiológica; Covid-19. 


\section{ABSTRACT}

This article analyzes the results of a survey carried out during the 2020 lockdown of high-level Spanish athletes (members of sporting federations and Olympians). Through the Emotional and Adaptive Reactions in Lockdown Questionnaire COVID-19 (REACOVID-19), distributed by federations and sports clubs to a non-probabilistic snowball sample, 2,831 athletes were interviewed. They were consulted about their habitats, family and leisure conditions during lockdown, training habits and behaviors, attitudes related to the motivation and organization of their training sessions, as well as the availability of materials, the monitoring of coaches and the information received by federations and sports clubs. Finally, they were also consulted about their position and how the suspension of the Tokyo 2020 Olympic Games has affected them. In addition, to paint a picture of these athletes' training situation in this context, the article exposes the difficulties and limitations of conducting a survey of this nature in a state of emergency.

Keywords: Federated, high level and Olympic sport; Survey; Lockdown; Epidemiological crisis; Covid-19.

\section{INTRODUCCIÓN ${ }^{1}$}

En el ámbito de las ciencias del deporte, se han realizado numerosos estudios sobre las actitudes, los hábitos y los comportamientos deportivos de la población española. Son bien conocidos los trabajos derivados de la Encuesta de Hábitos Deportivos en España (EHE), que realiza el Centro de Investigaciones Sociológicas (CIS) y el Instituto Nacional de Estadística (INE) desde el año 1980 de forma quinquenal. Desde la sociología del deporte se han realizado también investigaciones en materia de deporte de alto nivel y deporte olímpico (García Ferrando et al., 1996), especialmente trabajos de análisis de los perfiles y las carreras profesionales de los deportistas que se encuentran bajo el paraguas de estos niveles deportivos (Vilanova y Casado, 2000). Pero tradicionalmente la psicología del deporte ha dedicado los mayores esfuerzos al estudio de los aspectos que

1. Quisiéramos agradecer la ayuda el Dr. Manuel Trujillo Pérez Lanzac en la orientación de ideas para la elaboración del cuestionario en aras a recoger preguntas relacionadas con el aislamiento social. Este es Catedrático de Psiquiatría de la Universidad de Nueva York y Jefe de Psiquiatría del Hospital de Bellevue, donde fue responsable de la asistencia psicológica derivada de los efectos producidos por el atentado del 11S. Lideró el grupo de trabajo que desarrolló el primer protocolo de la Asociación Mundial de Psiquiatría para la asistencia Psicológica y Psiquiátrica en situaciones de catástrofes.

No quisiéramos dejar de manifestar que en la toma de las decisiones relativas a la definición de la muestra procedente de la encuesta de deportistas a analizar, fue determinante el asesoramiento y colaboración técnica del estadístico Juan Antonio Domínguez-Álvarez, Técnico de Investigación del Instituto de Estudios Sociales Avanzados (CSIC), experto en diseños estadísticos de campo y diseños de muestras y encuestas web. 
rodean la motivación, el estado anímico, las condiciones ambientales y emocionales de los deportistas de competición, sean estos federados, de alto nivel u olímpicos (Serpa y Castro, 2006; Cruz, 1992).

La población que desarrolla su actividad deportiva bajo el paraguas de lo que se entiende por "alto rendimiento", "alto nivel" y/o "profesional" representa un número limitado: 4.962 deportistas de alto rendimiento, 2.370 futbolistas profesionales, y otros tantos hombres y mujeres dedicados al baloncesto, tenis, balonmano y ciclismo, natación, remo, gimnasia, etc. Por tanto, es un número pequeño si se compara con los 25 millones de personas que, aproximadamente, realizan deporte amateur en España o, incluso, los 3.866.867 deportistas con credencial federativa que existen en nuestro país (MCD, 2020), y a los que calificaremos en este trabajo como "deportistas federados".

Los citados deportistas - en este y en sucesivos casos utilizamos el término "deportista" para referirnos tanto a hombres como a mujeres- ejercen un papel de enorme calado en el ámbito de la representación institucional y la identidad colectiva de federaciones y territorios, puesto que representan a nuestros clubes, ciudades y al país en las grandes competiciones. Hablamos de campeones mundiales y medallistas olímpicos, futbolistas, tenistas, ciclistas y deportistas de otras disciplinas, de primera división o división de honor, que en el tiempo de confinamiento y el momento posterior a la crisis sanitaria han sentido truncadas las expectativas de continuidad en sus trayectorias deportivas, e incluso muchos de ellos han quedado abocados al desempleo de la noche a la mañana.

El deporte federado y de alto nivel se realiza en el marco reglado de los clubes y federaciones deportivas, y en la participación en pruebas y competiciones nacionales e internacionales. En estos meses, estas pruebas y competiciones se han interrumpido o han experimentado importantes adaptaciones para su celebración, tanto las del deporte rey, el fútbol, como las de otros deportes tradicionales. A nivel internacional, pruebas deportivas señeras como la Champions (Liga de Campeones de la UEFA), la Fórmula 1, el Circuito Mundial de Motociclismo, el Tour de Francia o el Giro de Italia han sufrido la misma decepción y, contra todo pronóstico, incluso se han pospuesto los Juegos Olímpicos de Tokio 2020. Ante tal abrumadora realidad, podemos afirmar que el mundo del deporte de competición ha recibido un duro golpe en el año 2020.

Habría que situar en un primer plano a esos deportistas a los que nos hemos referido, que disponen de credencial de alto nivel, aproximadamente unos 4.962, verdaderos profesionales que cuentan con una beca del Consejo Superior de Deportes o de sus correspondientes federaciones deportivas y patrocinadores, con la que disfrutan de recursos y servicios humanos, alojamiento, medios técnicos, para su entrenamiento y excelencia en el deporte. Son deportistas que se proponen objetivos deportivos a corto, medio y largo plazo, e incluso que forman parte de equipos olímpicos para los Juegos Olímpicos de Tokio 2020, que ya será Tokio 2021. 
Durante los meses de confinamiento estuvieron entrenando en casa, sufriendo una gran inquietud por su futuro como deportistas, dado que el coste de oportunidad de la carrera deportiva es alto (a nivel personal, familiar, formativo, profesional fuera del terreno del deporte). La situación vivida durante el confinamiento fue de inquietud extrema, porque, con la salvedad de los futbolistas de primera división, todos los demás (especialmente, los de niveles de competición medios e inferiores) se encontraron en algunos casos en el paro, de la noche a la mañana; algunos de ellos cuentan con el deporte como único ingreso y actividad profesional. Especial mención requieren las mujeres deportistas profesionales, dado que su situación es completamente anómala, al no encontrarse en muchos casos sujetas a convenios laborales, por lo que durante el confinamiento se convirtieron en el grupo de deportistas más perjudicado.

No existen trabajos precedentes sobre la situación de los deportistas en contextos de aislamiento, pese a que somos conscientes de que hay deportistas de alto nivel y olímpicos que han padecido el aislamiento en contextos bélicos. Sin embargo, tras una revisión bibliográfica exhaustiva en las principales fuentes de referencia, no se han encontrado trabajos sobre este asunto. Las publicaciones con cierta relación son recientes y emanan de la presente crisis epidemiológica del coronavirus, teniendo que ver en su mayoría con el impacto emocional que ha causado sobre los deportistas de alto nivel y olímpicos (Ciddi y Yazgan, 2020; Clemente-Suárez et al. 2020; Da Ramos et al. 2020; Di Fronso et al. 2020; Guilherme et al. 2020; Jaenes et al. 2020; Jaenes et al. 2020a; Ozen, Koc y Aksoy, 2020; Reardon et al. 2020; Schinke et al. 2020).

Por lo tanto, este trabajo arroja luz justamente sobre la situación vivida (condiciones residenciales, ocio, horas de entrenamiento, etc.) por parte de los deportistas federados, de alto nivel y olímpicos, durante el período de confinamiento y estado de alarma vividos en España en la primavera de 2020. Por medio de una encuesta proporcionada a través de federaciones y clubes deportivos, con una muestra no probabilística de bola de nieve, fueron entrevistados 2.831 deportistas españoles. Se les solicitó información sobre sus características sociodemográficas y socioeconómicas (sexo, edad y nivel de instrucción), las condiciones del hábitat, familiares y ocio en que vivían el confinamiento, los hábitos y comportamientos de entrenamiento, actitudes relacionadas con la motivación y organización de sus entrenamientos, así como cuestiones de logística tales como los materiales disponibles, el seguimiento de entrenadores y la información recibida de federaciones y clubes deportivos durante el confinamiento. Por último, también se les consultó sobre su posición y el modo como les afectó personalmente la suspensión de los Juegos Olímpicos de Tokio 2020.

En el presente trabajo hemos circunscrito la muestra de explotación a 1.996 casos, explicando las razones y criterios de selección en el siguiente punto. Además, hemos explotado tan solo cuestiones relativas a comportamientos, información, opiniones y 
valoración de las circunstancias que rodearon la vida de estos deportistas durante el confinamiento; excluimos del presente análisis aquellas preguntas de escala Likert sobre conductas propias de la psicología clínica. Para concluir, hay que destacar el enorme reto que ha supuesto el recabar y explotar resultados de encuesta obtenidos a través de una de las pocas formas en que era posible durante el período de confinamiento y de estado de alarma, al haberse suspendido por completo todas las actividades económicas y profesionales no esenciales.

\section{LA CONTRASTACIÓN DE LA REALIDAD DEL DEPORTE DURANTE EL CONFINAMIENTO POR LA COVID-19 EN ESPAÑA. APROXIMACIÓN METODOLÓGICA}

Como consecuencia de la crisis epidemiológica mundial del SARS-CoV-2 (conocido como covid-19), el Gobierno de España, como otros muchos gobiernos nacionales, decidieron adoptar medidas inéditas de movilidad y distanciamiento social, que se tradujeron entre otras, inicialmente, en la más radical, esto es, el confinamiento en el hogar de todas aquellas personas que no ejercieran actividades esenciales (sanidad, educación, seguridad, alimentación, etc.) o para la estricta adquisición de alimentos y medicamentos, desde el 15 de marzo al 21 de junio de 2020.

En este contexto, como ya hemos descrito, se produjo una parálisis del deporte en España. Una situación inédita en la historia moderna que obligó a quienes lo practican a permanecer confinados en sus hogares durante casi dos meses, con la suspensión de todos los entrenamientos y pruebas deportivas, las grandes ligas y los campeonatos.

Ante esta coyuntura, un equipo de investigadores del ámbito de las ciencias sociales y del deporte (psicólogos y egresados de ciencias del deporte) — liderado por el Dr. Jaenesasumió la decisión estratégica de atender la evolución emocional y la adaptación a la situación del confinamiento de un grupo de deportistas de alto nivel. En el momento preliminar, se pensó exclusivamente en dar respuesta a ese cometido entre los remeros y remeras de la Federación Española de Remo que entrenan habitualmente en diferentes instalaciones de la geografía española. Al tener conocimiento la Consejería de Educación y Deportes de la Junta de Andalucía de la iniciativa planteada a través de la Unidad de Psicología del Deporte del Centro Andaluz de Medicina del Deporte (CAMD), desde esta administración se le solicitó extender dicho propósito para conocer la situación de los deportistas de alto nivel de otras disciplinas y federaciones. Igualmente, la Real Federación Española de Natación (RFEN), al tener conocimiento de esta iniciativa, pidió al investigador principal que permitiera a nadadores de las diferentes especialidades participar en dicha encuesta, por lo que se distribuyó la misma a través de clubes y entrenadores de todo el territorio español. Para ello, se ofreció a colaborar en el contacto de estos deportistas mediante el uso de sus bases de datos, algo que permitió llegar a una muestra de población de deportistas de 2.831 personas. 
De nuevo, lo que se planteaba inicialmente era una actividad dirigida a la atención de la evolución emocional y la adaptación a la situación del confinamiento de un grupo de deportistas de alto nivel - con el objetivo de establecer planes de intervención-y, por tanto, no una investigación clínica ni social en el estricto sentido del término. De ahí que su realización no quedara exenta de un conjunto de problemas metodológicos que no quisiéramos ocultar en este trabajo, sino más bien, a la postre, compartirlos y explicar a la vez qué medidas se han adoptado tanto en el trabajo de recogida de información, como en el tratamiento de los datos, para extraer conclusiones de carácter científicas, e invitar con ello a la reflexión sobre el uso de esta técnica en el futuro.

\subsection{Método y técnica para estudiar la realidad social desde el confinamiento}

Pocas opciones había para llegar a una población de deportistas que se encontraban confinados en casa por la crisis epidemiológica salvo mediante el uso de una encuesta telefónica o autoadministrada a través de Internet. Ciertamente, durante el confinamiento por la covid-19 en la primavera de 2020 existía un impedimento físico para realizar encuestas, entrevistas o trabajo etnográfico presencial. Por lo demás, como se ha apuntado, no se respondía con este trabajo a una investigación planificada, que por tanto dispusiese de recursos para realizar una encuesta telefónica, porque asistíamos a un acontecimiento histórico completamente imprevisto. Ni siquiera hubiera podido realizarse una encuesta telefónica, porque las actividades económicas y profesionales en España estaban suspendidas excepto las esenciales.

En ese marco, el equipo de investigadores decidió realizar una encuesta sobre el estado emocional, las condiciones materiales y los hábitos de entrenamiento, vividos por la población de deportistas de alto nivel en España durante el período de confinamiento. La encuesta se administró a través de Internet, desde la perspectiva en que se sitúa la definición de estas herramientas de recogida de información para Callegaro et al. (2015:5): "Una encuesta web es el modo de administración de encuestas que emplea cuestionarios informáticos autoadministrados, alojados en un servidor web conectado a Internet, al que los respondientes acceden a través de un navegador web". Dicha encuesta se diseñó y administró empleando la herramienta informática de cuestionarios Google Form. Se trata de una herramienta accesible y gratuita de la compañía Google que permite crear formularios para la obtención de información y recopilar los resultados en una base de datos de cálculo, generalmente la herramienta Excel del paquete informático Office de Microsoft. Es una herramienta segura, porque el acceso a dicha base de datos está limitada a los usuarios que administran el cuestionario, dado que la gestión de la misma está restringida a la cuenta personal a la que se vincula.

Las encuestas administradas a través de Internet son cada vez más habituales en el terreno de la investigación social, al encontrase y adquirir mayor relevancia estas en el uso ordinario de la vida social. No en vano, según el Instituto Nacional de Estadística 
(INE) - que realiza la Encuesta sobre Equipamiento y Uso de las Tecnologías de la Información y Comunicación en los Hogares-, mientras que en el año 2000 apenas un 14\% de la población española decía utilizar Internet, en el año 2019 la frecuencia de uso de este medio de comunicación alcanzaba ya al 91\%. Dado que han cambiado los modos de vida de la sociedad contemporánea, la cual pasa cada vez menos tiempo en casa, lo que hace difícil la realización de encuestas presenciales o a través de teléfonos fijos (que de la misma manera han ido siendo sustituidos por la telefonía móvil), el uso de la encuesta a través de Internet se ha ido extendiendo progresiva e intensamente.

Diferentes metodólogos españoles han analizado el uso de la encuesta a través de Internet en los últimos años (Díaz de Rada et al., 2019; Díaz de Rada y Domínguez, 2017; Díaz de Rada, 2011 y 2012). En esos trabajos, los autores ponen de manifiesto que "una visión retrospectiva de la evolución en la recogida de información mediante encuesta desvela que el cuestionario con ordenador (CAI) está sustituyendo paulatinamente a la encuesta tradicional presencial. Este tipo de cuestionarios administrados por ordenador, además de utilizar todos los tipos de preguntas disponibles (respuesta única, respuesta múltiple, respuesta textual o numérica, etc.), es más sencillo de administrar al llevar a cabo —de forma automática - "saltos" provocados por preguntas filtro, rutas complicadas, etc. No menos importante es que permite establecer reglas de consistencia que son comprobadas antes de grabar la información" (Díaz de Rada y Domínguez, 2017: 138).

Los resultados del estudio llevado a cabo por los citados autores - a partir de la investigación de Moscoso y Moyano (2010) a una muestra de población de 25.000 andaluces/as emigrantes en el exterior y residentes en 20 países de todos los continentes, entre los que se les ofreció responder a una encuesta auto-administrada mediante correo postal, de forma telefónica a través de un teléfono gratuito y mediante una versión web del cuestionario-, puso de manifiesto que "la realización de encuestas vía web reducen el tiempo del trabajo de campo, consiguen menor respuesta parcial y presentan un coste ligeramente inferior a la encuesta realizada por correo. La diferente estructura de costes de cada una permite concluir que, de haber obtenido el total de los cuestionarios online, la reducción de costes hubiera supuesto un 18,2\%" (Ibid.: 137). En efecto, los costes se reducen por la facilitad de implementar la encuesta, la informatización del cuestionario, la rapidez en el proceso de la recogida de datos y la gestión tanto de la planificación y seguimiento como de la participación geográfica y temporal.

En el caso de la investigación que atañe al presente trabajo, se han seguido algunas de las recomendaciones de los expertos en el empleo de este tipo de técnicas de recogida de información, como la definición del objetivo y el perfil de la muestra de población que será objeto de la investigación, la elección del diseño general de la encuesta y del cuestionario validado, y la delimitación del protocolo de contacto con los informantes. Pero otros elementos procedimentales que detallamos a continuación no han podido ser implementados por las circunstancias coyunturales aludidas. 
De partida, somos conscientes de que Google Form no es una herramienta idónea para una encuesta web y, por ello, no queremos dejar de poner de manifiesto nuestra actitud de cautela sobre algunas de las dificultades y problemáticas que genera. En efecto, Google Forms es un programa diseñado para proporcionar a sus usuarios una forma ágil de incorporar formularios y sondeos sencillos en sus páginas web. Se trata de herramientas que no están pensadas específicamente para la gestión de encuestas web científicas, por lo que suelen tener bastantes limitaciones a la hora de incorporar elementos básicos de una encuesta propiamente científica, como los controles de flujo de la entrevista (saltos y filtros) o los controles de consistencia en las respuestas. Además, la herramienta tampoco proporciona opciones para la gestión de la muestra de la misma encuesta, algo esencial que sí deberían hacer los programas de creación y gestión de encuestas web científicas (Díaz de Rada et al., 2019: 37). A las anteriores problemáticas hay que añadir la dificultad para controlar duplicados - aunque en este estudio eso no se haya producido, porque solo se daba una opción de respuesta por cada usuario- y controles de calidad gracias a paradatos y cookies como la duración de cada cuestionario o la localización geográfica.

Por lo anterior, quisiéramos dejar claro, desde la honestidad académica, que este trabajo de investigación está sujeto a limitaciones y déficit de control de validez y fiabilidad, tanto específicamente en la administración de la encuesta como, en concreto, por los impedimentos circunstanciales que acompañaron a la realización del trabajo de campo durante el estado de alarma. Pero sin la capacidad de actuar con agilidad de otra manera, como consecuencia de la suspensión de la actividad económica en España, que impedía poner en contacto a equipos de investigación multidisciplinares más amplios (psicólogos, científicos del deporte, sociólogos, informáticos y estadísticos), al no estar autorizada su actividad profesional, el procedimiento de ejecución de la encuesta de forma óptima era muy difícil de implementar. Otra situación diferente es la que atañe al resto de fases del trabajo de investigación, que detallamos a continuación, como es el diseño del cuestionario de recogida de información y la selección de la muestra de población que ha sido objeto del tratamiento estadístico, para desarrollar inferencias científicas.

Con todo, para la realización del presente trabajo se diseñó un instrumento ad hoc en Google Drive, cuya validez y fiabilidad está documentada de acuerdo con las recomendaciones de Díaz de Rada, Domínguez-Álvarez y Pasadas del Amo (2019) y que, dada la situación de confinamiento, era el más adecuado.

\subsection{El cuestionario "Reacciones emocionales y adaptativas en el confinamiento COVID-19 (REACOVID-19)”}

Para el diseño del cuestionario, el equipo de investigación consensuó la herramienta partiendo, inicialmente, de la búsqueda de referencias en MEDLINE y Google Scholar, tratando de localizar ejemplos empleados en estudios con personas en situación de 
aislamiento. Asimismo, se contactó con un experto en este tipo de situaciones, el Dr. Manuel Trujillo Pérez Lanzac.

Resultaron de utilidad tanto las recomendaciones proporcionadas por el citado Doctor, como, por otro lado, la versión corta del cuestionario POMS de Andrade et al. (2013) para deportistas adultos y población general, al que se añadieron preguntas sobre el impacto del confinamiento en esferas como el sueño, la concentración y los estados de ánimo. La construcción del mismo tenía como propósito saber más acerca de su adaptación psicológica, cognitiva y emocional.

Una vez redactadas las preguntas y las opciones de respuesta, el equipo de investigación expuso el cuestionario a la discusión académica colegida, tras la que se llegó a un acuerdo sobre la versión definitiva, concretada en el cuestionario denominado Reacciones emocionales y adaptativas en el confinamiento COVID-19 (REACOVID-19) (Jaenes et al., 2020). En total, el cuestionario se compone de 43 preguntas, abiertas, cerradas y categorizadas, de identificación, hecho, acción, opinión e información y de batería.

- Entre las preguntas, existe un primer bloque de cuestiones sociodemográficas, en donde se solicita información sobre su edad, sexo y nivel de instrucción; también se solicita información relativa a la Comunidad Autónoma y el país de residencia.

- En un segundo bloque se requiere a los participantes información sobre aspectos condicionantes de sus vidas durante el confinamiento: tamaño de la residencia, disfrute de espacios abiertos en la misma, situación laboral familiar y actividades de ocio.

- En el tercero, tras solicitar información sobre el tipo de práctica que realiza y el nivel de dedicación, se recaban datos sobre hábitos de entrenamiento durante el periodo de confinamiento. También se les preguntó si disponían de material para entrenar adecuadamente, el seguimiento de los entrenadores, la información recibida por parte de los clubes y federaciones, etc.

- En un cuarto bloque, se preguntaba - solo a los deportistas olímpicos- su opinión sobre la suspensión de los Juegos Olímpicos y el efecto que ello tendría en su carrera deportiva.

- Por último, un quinto bloque de preguntas estaba dirigido a recopilar datos sobre la adaptación psicológica, cognitiva y emocional, ante la situación de confinamiento que se vivía en España en ese momento.

\subsection{Modo de administración de la encuesta y diseño muestral}

Una vez que el cuestionario había quedado diseñado y validado, el equipo de investigación lo probó con un pre-test entre diez deportistas de alto nivel, cinco entrenadores y tres psicólogos del deporte, para garantizar que las preguntas del cuestionario se entendían perfectamente y no daban lugar a confusión. Desde los primeros días del confinamiento, 
el cuestionario comenzó a difundirse entre deportistas, especialmente a través de la lista de contactos de la Federación Española de Remo, la Federación Española de Natación y el Centro Andaluz de Medicina del Deporte (CAMD) de la Consejería de Educación y Deportes de la Junta de Andalucía.

Desde ese planteamiento, conviene aclarar que lo que se ha obtenido ha sido una muestra no probabilística, a partir de un muestreo por conveniencia, conocido como bola de nieve. En este caso, al ser tan escasa la población de deportistas de alto nivel, lo que hace que la muestra tienda a ser agrupada, este tipo de diseño muestral está justificado. En condiciones normales, la población hubiese podido resultar más fácilmente localizable, si las estructuras organizativas de federaciones y clubes no se hubieran encontrado sujetas a las mismas condiciones de restricción que el equipo investigador, debido al confinamiento y la suspensión de las actividades económicas no esenciales.

El inicio de la administración de la encuesta tuvo lugar el 23 de abril y concluyó el 25 de mayo. En ese tiempo se logró una participación de 2.831 personas. Pero, dado que no había capacidad de controlar y cribar el perfil de los participantes, al analizar los datos se comprobó que muchos de esos informantes no se correspondían con la población diana. A pesar de la gran cantidad de sesgos de una muestra de este tipo, juega a su favor disponer de una población cautiva, en el sentido, no del confinamiento, sino de un censo al que se accede fácilmente a través de los clubes, que en este caso habían colaborado vía federaciones - en la búsqueda de informantes.

En consecuencia, desde el punto de vista de la muestra, algunos de los posibles sesgos detectados son los siguientes:

a) Sesgos de disciplina deportiva. La muestra total obtenida no representa por igual a todas las disciplinas deportivas, sino que la mayor parte de los casos están representados por deportistas cuyas disciplinas se encuentran adscritas a las federaciones que colaboraron con mayor compromiso a la difusión de la encuesta, en concreto, natación y remo, por lo que estas representan el $71 \%$ de la muestra (1.996 casos).

b) Sesgos de nivel de práctica. De la misma manera, la muestra alcanzada a través de la encuesta recoge una amplitud de casos que no cumplen el propósito inicial del trabajo de investigación, pues participa una diversidad mayor de perfiles de deportistas. Esto tiene que ver con las dificultades advertidas inicialmente, tanto las descritas para el uso de la herramienta Google Forms, como las que se desprenden del tipo de muestreo no probabilístico de bola de nieve. Con lo cual, del conjunto de la muestra de casos obtenida, menos de la mitad (el 47\%, 1.325 casos) afirma competir a nivel nacional e internacional (1.082 casos y 243 casos, respectivamente). De todos estos casos, 158 serían deportistas de alto rendimiento y, de ellos, 88 serían olímpicos, el $8 \%$ y el 4\%, respectivamente. 
c) Sesgos de género. La distribución de género tampoco está proporcionalmente representada en la muestra general. Del total de la muestra, que -recordémosloeran 2.831 casos-, el $51 \%$ son mujeres ( 1.456 casos); es una cifra que lamentablemente dista de la realidad, pues en la práctica la población de mujeres deportistas es entre $10 \mathrm{y}$ 15 puntos inferior a la de hombres deportistas - según la disciplina deportiva y más aún en el terreno federado-, aunque, paradójicamente, puede ser mayor el número de mujeres que de hombres, según la disciplina deportiva, en el alto nivel y el deporte olímpico.

En cuanto a los restantes datos sociodemográficos de la muestra resultante, la encuesta proporciona la siguiente distribución por edad y nivel de estudios.

- En el caso de la edad, dadas las características de la población, es normal que el grueso de la muestra tenga menos de 24 años (el 78\%), siendo la edad media 21,33 años. Si atendemos a su diferenciación según deportistas federados, de alto nivel y olímpicos, la edad media de estos tan solo sobresale entre los deportistas de alto nivel (26,04 años). La edad en la muestra también varía entre hombres y mujeres, siendo más elevada en los hombres (22,98 años de media) que en las mujeres (19,27 años de media).

- En cuanto a nivel de estudios, y teniendo presente la edad media de la muestra de población, es igual o inferior a bachillerato. El $47 \%$ tiene estudios primarios, de ESO o equivalente, el $20 \%$ bachillerato o equivalente y el $19 \%$ estudios universitarios. La situación cambia entre los deportistas de alto nivel, que tienen en mayor proporción estudios de bachillerato o equivalente y estudios universitarios ( $35 \%$ y $32 \%$, respectivamente). En el mismo orden se sitúan los deportistas olímpicos, entre los que un $42 \%$ cuenta con estudios de bachillerato o equivalente y un $32 \%$ con estudios universitarios. Si atendemos al sexo, predomina un mayor porcentaje de quienes tan solo cuentan con estudios primarios, ESO o equivalentes, entre las mujeres que entre los hombres (54\% y $41 \%$, respectivamente), en correspondencia también con su edad media.

\subsection{Tratamiento de los datos}

Los datos obtenidos a través de la administración de la encuesta se han tratado siguiendo un escrupuloso proceso de delimitación de la muestra resultante. Una vez analizados los detalles de dicha muestra, las decisiones estratégicas adoptadas para el aprovechamiento de los resultados del trabajo fueron las que se indican a continuación. También queremos subrayar que las decisiones aquí adoptadas están dirigidas a compensar o atajar las debilidades asociadas a las derivadas del método de recogida de información, de tal manera que pueda establecerse un mecanismo de representatividad válido y fiable en la explotación de los resultados de la muestra definitivamente seleccionada.

- En primer lugar, desde el punto de vista de la disciplina deportiva de los participantes en la encuesta, se decide limitar la explotación de la muestra a los casos de quienes afirman practicar natación y remo en sus diversas modalidades. De esta forma, nos 
quedaríamos con una muestra general de 1.996 casos (el 71\% del total). De esta muestra, el 84\% (1.683 casos) representaría a 'natación' y el 16\% (313 casos) representaría a 'remo' en sus diferentes variedades.

- En segundo lugar, en lo que concierne al nivel de práctica entre los casos de natación y remo, decidimos explotar de manera diferenciada tres perfiles: uno, el de los deportistas federados que compiten (a nivel autonómico y nacional), a los que calificaremos de 'deportistas federados'; otro, el de los 'deportistas de alto nivel' (que compiten a nivel internacional), de los que extraemos un subgrupo que representa casi la mitad y que calificamos como 'deportistas olímpicos'. Entre los primeros, la muestra estará representada por 1.815 casos; entre los segundos, por 158 casos (8\%), y entre los terceros por 88 casos (4\%). La distribución de los casos por disciplinas deportivas se recoge en las tablas 1 a 4 .

- En tercer lugar, en lo que atañe al género de los casos analizados en la muestra definitivamente seleccionada, el porcentaje es más aproximado a la realidad, reflejando un 55\% de hombres y un $45 \%$ de mujeres. Al igual que en el segmento anterior, la distribución por disciplinas deportivas y calificación del nivel de práctica se presentan en las siguientes tablas.

\begin{tabular}{|l|c|l|l|l|l|}
\hline \multirow{2}{*}{$\begin{array}{l}\text { Ámbito de } \\
\text { Competición }\end{array}$} & \multicolumn{2}{|c|}{ Natación } & \multicolumn{2}{c|}{ Remo } & \multirow{2}{*}{ Total } \\
\cline { 2 - 5 } & Hombres & Mujeres & Hombres & Mujeres & \\
\hline Autonómico & 518 & 441 & 45 & 29 & 1.033 \\
\hline Nacional & 313 & 271 & 136 & 61 & 781 \\
\hline Internacional & 57 & 61 & 23 & 17 & 158 \\
\hline $\begin{array}{l}\text { Master/ } \\
\text { Veteranos }\end{array}$ & 15 & 8 & 1 & 0 & 24 \\
\hline Total & 903 & 781 & 205 & 107 & 1.996 \\
\hline
\end{tabular}

Tabla 1. Muestra total seleccionada de la investigación para la explotación y análisis. Fuente: Elaboración propia. 


\begin{tabular}{|c|c|c|c|c|c|c|}
\hline \multicolumn{6}{|c|}{ Estadísticas Oficiales del Consejo Superior de Deportes (CSD) (año 2019) } \\
\hline & \multicolumn{2}{|c|}{ Hombres } & \multicolumn{2}{c|}{ Mujeres } & Total \\
\hline Natación & 37.276 & $54 \%$ & 32.207 & $46 \%$ & 69.483 \\
\hline Remo & 8.452 & $65 \%$ & 4.477 & $35 \%$ & 12.929 \\
\hline \multicolumn{7}{|c|}{ Muestra del estudio } \\
\hline Hombres & \multicolumn{2}{c|}{ Mujeres } & Total \\
\hline Natación & 831 & $54 \%$ & 712 & $46 \%$ & 1543 \\
\hline Remo & 181 & $67 \%$ & 91 & $33 \%$ & 272 \\
\hline
\end{tabular}

Tabla 2. Comparación de estadísticas oficiales de deportistas federados y muestra del estudio. Fuente: Elaboración propia.

\begin{tabular}{|c|c|c|c|c|c|c|}
\hline \multicolumn{5}{|c|}{ Estadísticas Oficiales del Consejo Superior de Deportes (CSD) (año 2019) } \\
\hline \multirow{2}{*}{ Hombres } & \multicolumn{2}{|c|}{ Mujeres } & Total \\
\hline Natación & 131 & $40 \%$ & 200 & $60 \%$ & 331 \\
\hline Remo & 71 & $80 \%$ & 18 & $20 \%$ & 89 \\
\hline \multirow{7}{*}{ Hombres } & \multicolumn{2}{|c|}{ Muestra del estudio } & Tujeres \\
\hline Natación & 57 & $48 \%$ & 61 & $52 \%$ & 118 \\
\hline Remo & 23 & $57 \%$ & 17 & $43 \%$ & 40 \\
\hline
\end{tabular}

Tabla 3. Comparación de estadísticas oficiales de Deportistas de Alto Rendimiento (D.A.R.) y muestra del estudio. Fuente: Elaboración propia. 


\begin{tabular}{|c|c|c|c|c|c|}
\hline \multicolumn{6}{|c|}{ Estadísticas Oficiales del Consejo Superior de Deportes (CSD) (año 2019) } \\
\hline & \multicolumn{2}{|c|}{ Hombres } & \multicolumn{2}{|c|}{ Mujeres } & \multirow{2}{*}{$\begin{array}{c}\text { Total } \\
66\end{array}$} \\
\hline Natación & 22 & $33 \%$ & 44 & $77 \%$ & \\
\hline Remo & 11 & $50 \%$ & 12 & $50 \%$ & 23 \\
\hline \multicolumn{6}{|c|}{ Muestra del estudio } \\
\hline & \multicolumn{2}{|c|}{ Hombres } & \multicolumn{2}{|c|}{ Mujeres } & Total \\
\hline Natación & 32 & $50 \%$ & 33 & $50 \%$ & 65 \\
\hline Remo & 16 & $70 \%$ & 7 & $30 \%$ & 23 \\
\hline
\end{tabular}

Tabla 4. Comparación de estadísticas oficiales de Deportistas Olímpicos (D.O.) y muestra del estudio. Fuente: Elaboración propia.

Una vez definida la base de datos válida para la explotación de resultados, convertimos su fuente original ".xml", propia del programa de hojas de cálculo Excel, a una fuente "sav" para poder adaptarla al programa estadístico SPSS (Statistical Package for Social Sciences). Los análisis realizados para la explotación de resultados a través de este programa informático en el presente trabajo son exclusivamente de estadística descriptiva, tales como tablas de frecuencia y tablas cruzadas.

\section{RESULTADOS}

\subsection{Condiciones materiales y sociales de los deportistas durante el confinamiento}

Las condiciones materiales y sociales vividas por los deportistas durante el confinamiento se han medido con diferentes variables. En primer lugar, en la encuesta se les ha consultado acerca de las condiciones residenciales en que se encontraban durante el confinamiento, en particular, sobre los metros cuadrados de la vivienda en la que pasaron este aislamiento 
y la disponibilidad o no de terraza o jardín —si bien no se les preguntó sobre el número de personas que comparten la vivienda. En segundo lugar, se les preguntó acerca de sus comportamientos en relación al cumplimiento de la normativa de aislamiento exigida por el gobierno durante el confinamiento. En tercer lugar, se les pidió información acerca de la situación laboral vivida por sus familiares a raíz de la crisis epidemiológica, en concreto, si alguno de sus familiares había perdido el trabajo. Y, en cuarto lugar, se quiso saber qué actividades realizaron en ese período para combatir el aburrimiento.

En relación al tamaño y la disponibilidad de espacios abiertos (terraza o jardín) de los deportistas durante el confinamiento, el grueso de la población consultada informa que lo pasaron en viviendas de entre $70 \mathrm{~m}^{2}$ y $120 \mathrm{~m}^{2}$ (ver gráfico 1). Tan solo 1 de cada 10 personas encuestadas pasó este período en viviendas de menos de $70 \mathrm{~m}^{2} \mathrm{y}$ una cuarta parte lo hizo en viviendas de más de $120 \mathrm{~m}^{2}$. En cuanto al disfrute de terraza o jardín exterior al lugar del confinamiento, 6 de cada 10 deportistas consultados afirman haber dispuesto de estos espacios.

En atención a las características de los deportistas, la situación no cambia mucho en lo que atañe a la dimensión de las viviendas donde pasaron su confinamiento, pero sí lo hace en lo que concierne al disfrute de jardín o terraza exterior a la vivienda, siendo más elevado en el caso de los deportistas de alto nivel y deportistas olímpicos que en el de la muestra general de deportistas federados (ver gráfico 2). Esta situación habrá contribuido a poder disfrutar de mejores condiciones para mantener un mínimo nivel de entrenamiento en esos casos.

En otro orden de cuestiones, también se consultó a estos deportistas si habían respetado el aislamiento social en casa durante el primer estado de alarma. La práctica totalidad de los deportistas respondió haber respetado mucho o bastante el confinamiento (99\%), afirmando el 57\% que no salió de casa ni siquiera cuando se dio permiso para salir con los hijos menores a partir del 26 de abril. Además, la mayoría (el 79\%) opina que era necesario respetar la cuarentena. Todo ello demuestra que, bien por responsabilidad, bien por garantizar su salud para con sus intereses deportivos, la mayoría de estos deportistas cumplió el confinamiento de manera convencida en sus domicilios. En este aspecto, no hay diferencias significativas marcadas por la edad o el sexo de las personas entrevistadas. En el caso de los deportistas de alto nivel y olímpicos, fueron muchos más los que no salieron de casa después del 26 de abril ( $88 \%$ y 67\%, respectivamente) (ver gráfico 3). Cabe la posibilidad de que esta reacción por parte de los citados deportistas tenga que ver con el temor a contagiarse por el covid-19, lo que pudiera haber afectado a la buena continuidad de sus carreras deportivas. 

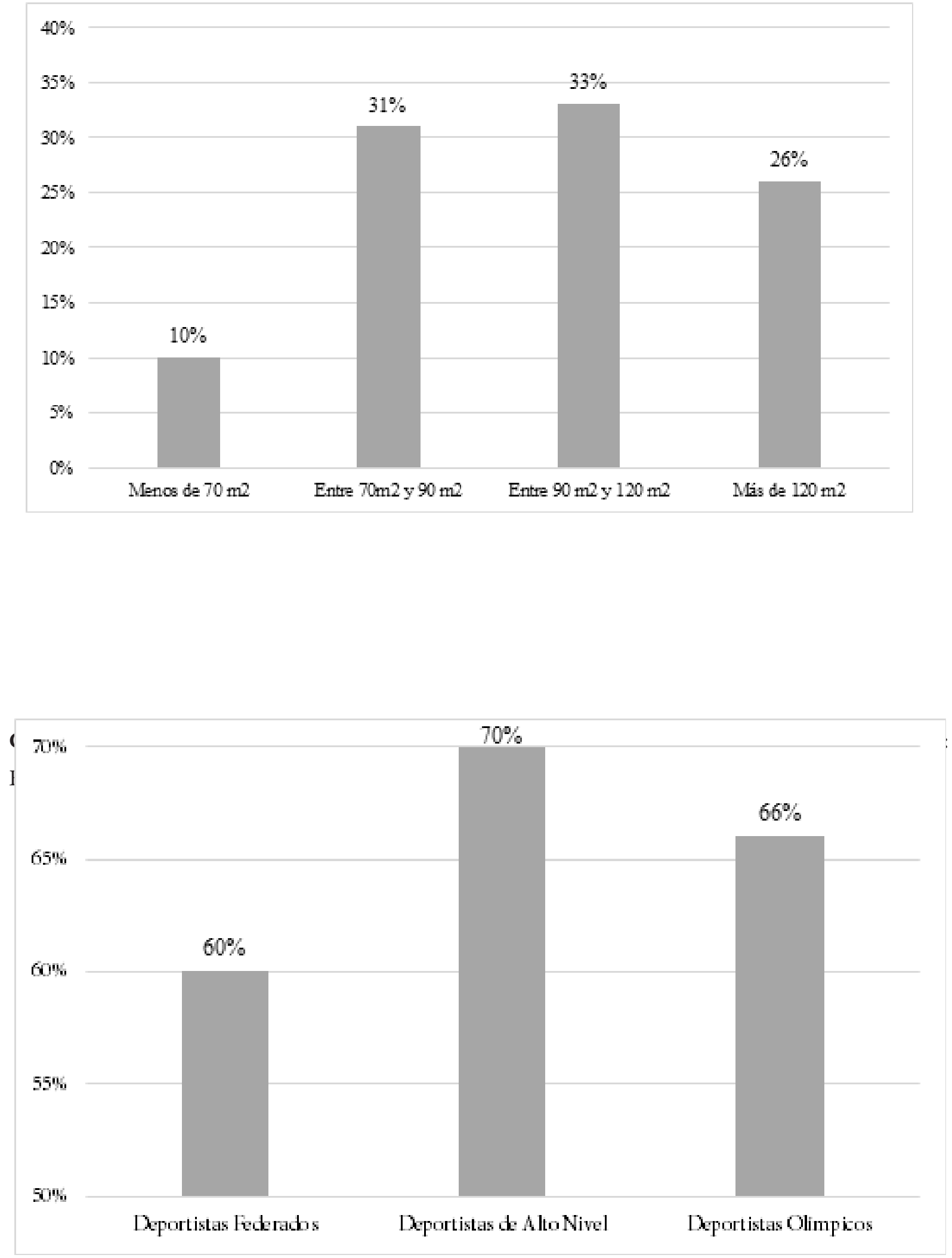

Gráfico 2. Disfrute de jardín y terraza exterior durante el confinamiento, según el perfil de los deportistas. Respuesta: SÍ. Fuente: Elaboración propia. 


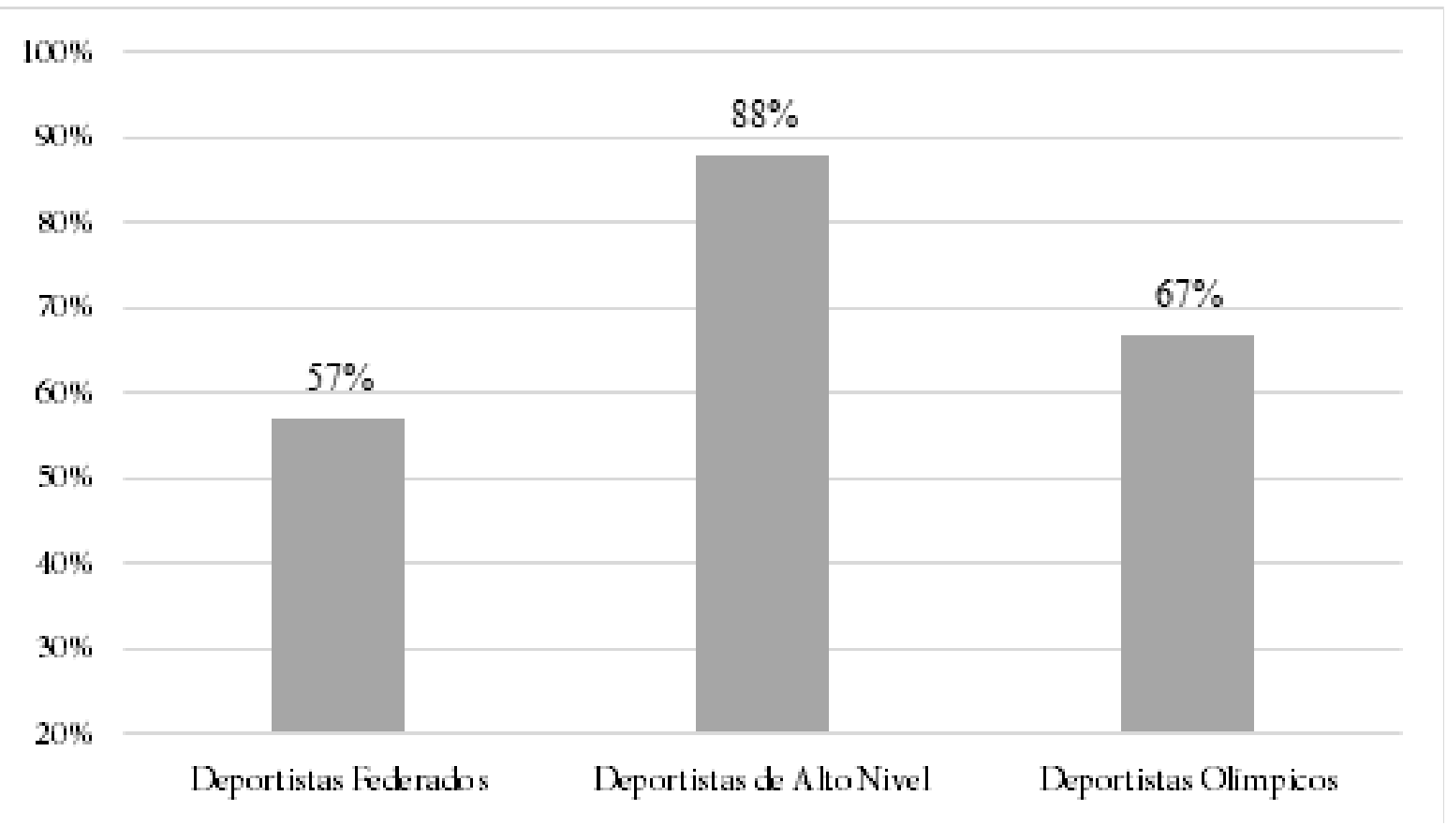

Gráfico 3. ¿Ha salido a la calle desde que se dio permiso para que los niños pudieran salir (26 de abril)? Respuesta: NO. Fuente: Elaboración propia.

En el análisis de las condiciones materiales y sociales de los deportistas durante el confinamiento se ha tenido en cuenta también la situación laboral de los familiares a raíz de la crisis epidemiológica, por considerar que esta podría influir en su estado anímico. Los resultados muestran que el $32 \%$ de los deportistas tienen familiares que han perdido el trabajo, siendo algo mayor el porcentaje de los deportistas olímpicos que se encuentran en esta situación (37\%).

Para finalizar este apartado, en relación a las principales actividades realizadas para combatir el aburrimiento durante el confinamiento, sobresalen las relacionadas con el uso de tecnologías digitales y la comunicación: las videoconferencias o llamadas a amigos o familiares (el 95\%), escuchar música (93\%), hacer uso de las redes sociales (89\%), ver la televisión (89\%) o los videojuegos (64\%). Otras actividades ocuparon a un menor porcentaje de personas, como estudiar (76\%), lectura (64\%) o aprender algo nuevo (61\%). Un menor número de personas aún ocupó el tiempo en técnicas de relajación (48\%), yoga (27\%), mindfulness (16\%), cocinar (15\%) o dibujar/pintar (5\%). 


\begin{tabular}{|c|c|c|c|}
\hline & $\begin{array}{l}\text { Deportistas } \\
\text { Federados }\end{array}$ & $\begin{array}{c}\text { Deportistas de Alto } \\
\text { Nivel }\end{array}$ & $\begin{array}{c}\text { Deportistas } \\
\text { Olímpicos }\end{array}$ \\
\hline $\begin{array}{l}\text { Videoconferencias } \\
\text { con familiares y } \\
\text { amigos }\end{array}$ & $95 \%$ & $92 \%$ & $93 \%$ \\
\hline Escuchar Música & $93 \%$ & $95 \%$ & $93 \%$ \\
\hline Ver la televisión & $89 \%$ & $85 \%$ & $86 \%$ \\
\hline Redes Sociales & $89 \%$ & $94 \%$ & $95 \%$ \\
\hline Estudiar & $76 \%$ & $86 \%$ & $89 \%$ \\
\hline Lectura & $64 \%$ & $76 \%$ & $69 \%$ \\
\hline Videojuegos & $64 \%$ & $53 \%$ & $58 \%$ \\
\hline $\begin{array}{l}\text { Aprender algo } \\
\text { nuevo }\end{array}$ & $61 \%$ & $69 \%$ & $72 \%$ \\
\hline Relajación & $48 \%$ & $57 \%$ & $62 \%$ \\
\hline Yoga & $27 \%$ & $42 \%$ & $44 \%$ \\
\hline Mindfulness & $16 \%$ & $28 \%$ & $32 \%$ \\
\hline Cocinar & $15 \%$ & $21 \%$ & $27 \%$ \\
\hline
\end{tabular}

Tabla 5. Durante el confinamiento, para salir del aburrimiento, ¿'ha utilizado alguno de estos métodos? Fuente: Elaboración propia.

Si atendemos a la variable sexo, se comprueban diferencias notables entre hombres y mujeres deportistas en la realización de algunas de estas actividades para combatir el tedio durante el confinamiento. 
- Los hombres ocuparon mucho más que las mujeres su tiempo en ver la televisión ( $54 \%$ y $46 \%$, respectivamente), en las redes sociales (55\% y $45 \%$, respectivamente), en los videojuegos (69\% y $31 \%$, respectivamente), en las videoconferencias (54\% y $46 \%$, respectivamente) y en escuchar música ( $54 \%$ y $46 \%$ ) o la radio (59\% y $41 \%$ ).

- Las mujeres ocuparon más su tiempo que los hombres en cocinar (66\% y $34 \%$, respectivamente), dibujar/pintar ( $79 \%$ y $21 \%$, respectivamente), yoga (66\% y $34 \%$, respectivamente), mindfulness ( $57 \%$ y $43 \%$, respectivamente) e idiomas ( $66 \%$ y $34 \%$, respectivamente).

De acuerdo con los perfiles de deportistas, existen diferencias significativas en la elección de las actividades dirigidas a hacer frente a la frustración durante el confinamiento. Entre los deportistas de alto nivel y olímpicos, la frecuencia de dedicación a la lectura, estudiar, relajación, mindfulness, yoga, aprender algo nuevo y cocinar es mucho mayor que entre el resto de deportistas federados. Entre estos últimos, en cambio, es mucho mayor el porcentaje de quienes dedicaron su tiempo para combatir el aburrimiento a los videojuegos. En lo que atañe al resto de actividades, existen mayores similitudes de frecuencia de realización.

\subsection{Situación, comportamientos y actitudes relacionadas con el deporte durante el confinamiento}

En relación a los hábitos y comportamientos de entrenamiento de los deportistas encuestados, los resultados muestran que estos mantuvieron una media de entrenamiento semanal de 5-7 horas. Algo que denota que, más allá de las dificultades derivadas del aislamiento social en el hogar como consecuencia de la crisis epidemiológica, quienes practican deporte federado tienen la capacidad de encontrar fórmulas con las que mantener sus hábitos de actividad física y deportiva. En el caso de los deportistas de alto nivel, la media de horas de entrenamiento semanal aumentaba hasta las 8-10 e incluso algo mayor era la media de horas de entrenamiento semanal entre deportistas olímpicos (11-13 horas).

Pese a las mencionadas medias, los datos evidencian que había deportistas que las superaban con creces, especialmente los de alto nivel y los olímpicos, entre los que un $21 \%$ y un $31 \%$ respectivamente entrenaron más de 16 horas semanales durante el confinamiento. En relación al número de horas de entrenamiento, no se observan diferencias entre hombres y mujeres. Sí se observan unas leves diferencias según el tipo de disciplina practicada (natación o remo), siendo la media de los deportistas de remo ligeramente mayor que la de natación (8-10 horas en el caso de los deportistas de remo, frente a las 5-7 horas en el caso de los deportistas de natación) (ver tabla 6). 


\begin{tabular}{|l|c|c|c|}
\hline \multirow{2}{*}{$\begin{array}{c}\text { Frecuencia de } \\
\text { entrenamiento }\end{array}$} & $\begin{array}{c}|c| \\
\text { Deportistas } \\
\text { Federados }\end{array}$ & $\begin{array}{c}\text { Deportistas de } \\
\text { Alto Nivel }\end{array}$ & $\begin{array}{c}\text { Deportistas } \\
\text { Olímpicos }\end{array}$ \\
\cline { 2 - 4 } Menos de 5 horas & $24 \%$ & $9 \%$ & $0 \%$ \\
\hline 5 a 7 horas & $34 \%$ & $23 \%$ & $24 \%$ \\
\hline 8 a 10 horas & $20 \%$ & $21 \%$ & $16 \%$ \\
\hline 11 a 13 horas & $12 \%$ & $20 \%$ & $25 \%$ \\
\hline 14 a 16 horas & $6 \%$ & $6 \%$ & $5 \%$ \\
\hline Más de 16 horas & $4 \%$ & $21 \%$ & $31 \%$ \\
\hline Media & $5-7$ & $8-10$ & $11-13$ \\
\hline
\end{tabular}

Tabla 6. Horas de entrenamiento semanal. Fuente: Elaboración propia.

Con respecto a las condiciones logísticas con las que los deportistas encuestados se encontraron durante el confinamiento, la encuesta proporciona información en relación al material del que disponían para ello, así como el contacto y seguimiento de aquellos por parte del entrenador (en su caso) y la información proporcionada por las federaciones y clubes deportivos.

En relación al material disponible para garantizar el entrenamiento durante el confinamiento, entre 6 y 7 de cada diez deportistas federados (el 65\%) contaban con "poco" o "nada" de material adecuado para ello (ver gráfico 4), siendo las condiciones similares tanto en hombres como en mujeres. Igualmente, el material disponible para el entrenamiento en ese contexto era similar tanto entre deportistas federados como entre deportistas de alto nivel y deportistas olímpicos. 


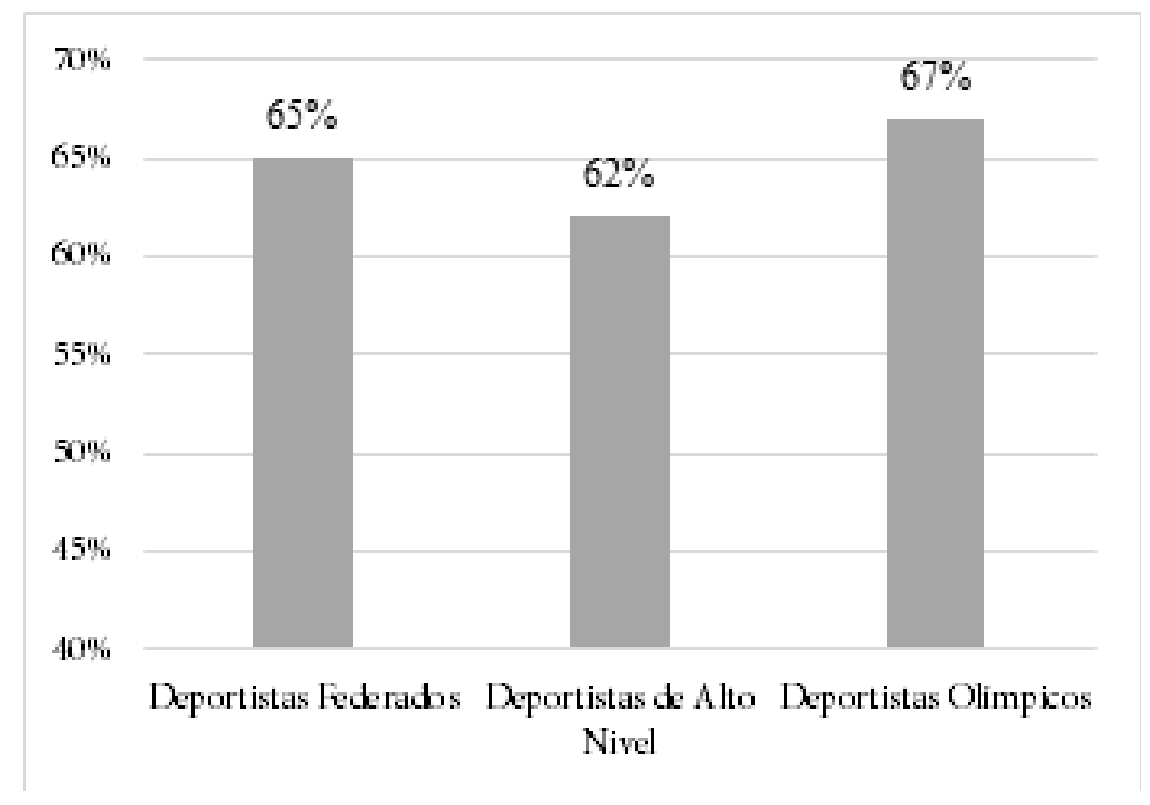

Gráfico 4. ¿Tenías material para entrenar adecuadamente? Respuesta: POCO; NADA. Fuente: Elaboración propia.

En lo que concierne al seguimiento por parte de los entrenadores de las sesiones de entreno, en general se confirma que 6 de cada 10 deportistas contaron con "bastante" o "mucho" apoyo. Ahora bien, existen algunas diferencias (ver tabla 7). Si los deportistas eran tan solo federados, contaron con esa ayuda el $58 \%$, pero si se trataba de deportistas de alto nivel y deportistas olímpicos, resultó mayor el porcentaje de los que contaron con ese apoyo (el 64\% en ambos casos). Sin embargo, mayores y realmente llamativas son las diferencias que se observan entre los deportistas hombres y mujeres que disfrutaron con el seguimiento de sus entrenadores ( $53 \%$ y $64 \%$, respectivamente). Más relevantes incluso son las diferencias en el porcentaje de quienes contaron con esa ayuda según el tipo de deporte practicado (remo o natación) (69\% y 56\%, respectivamente).

\begin{tabular}{|c|c|c|}
\hline \multicolumn{3}{|c|}{ Variables } \\
\hline Nivel Deportivo & Sexo & Disciplina Deportiva \\
\hline Federados: $58 \%$ & Hombres: $53 \%$ & Natación: $56 \%$ \\
Alto Nivel: $64 \%$ & Mujeres: $64 \%$ & Remo: $69 \%$ \\
Olímpicos: $64 \%$ & & \\
\hline
\end{tabular}

Tabla 7. Seguimiento por parte de los entrenadores para los entrenamientos durante el confinamiento. Respuesta: MUCHO; BASTANTE. Fuente: Elaboración propia. 
Aparte del seguimiento de los entrenos, a los deportistas encuestados se les preguntó también por la información recibida durante el confinamiento por parte de federaciones y clubes. Al preguntarles si la federación les informaba sobre la evolución de la situación en su deporte, la mayoría de deportistas contestaron que recibieron poca o nada de información por aquellas (ver tabla 8). Cierto es que existen algunas diferencias de frecuencia en esta respuesta de acuerdo con las categorías, pues resulta mayor la frecuencia de dicha respuesta entre los deportistas federados (63\%) que entre los de alto nivel (55\%) y los olímpicos (53\%). También es significativa la diferencia en la frecuencia de tal respuesta entre quienes practican natación y remo, un $64 \%$ y un 53\% respectivamente, algo que hace pensar que ambas federaciones actuaron de forma diferente.

Hay que decir a este respecto que es normal que en las federaciones no se informase durante el periodo de confinamiento de forma óptima a los deportistas sobre la situación de su deporte, porque durante aquel momento las federaciones tampoco disponían de la suficiente información, o esta era contradictoria a menudo, según se recibiera desde el gobierno de la nación, a través del Consejo Superior de Deportes, o desde las direcciones generales de deporte de los gobiernos autonómicos. Además, al darse una situación de estado de alarma que limitaba toda actividad económica en el país, los responsables de las federaciones deportivas no podían ejercer su actividad y, por tanto, informar, lo que explicaría en su conjunto la falta de información de las federaciones - a juicio de los encuestados-durante el confinamiento.

Sin embargo, este juicio contrasta con el que emiten para referirse a otra pregunta similar: “'Tu club o equipo te informaba sobre la evolución de la situación en tu deporte (si hay o no competición, si se suspende algún evento deportivo, etc.)?”. Ante esta pregunta, la respuesta es inversa, siendo mayoritaria la respuesta positiva ("mucho" o "bastante"), que representaba a 7 de cada 10 personas encuestadas (ver tabla 8). Pero en este caso son los deportistas federados - más que los deportistas de alto nivel y olímpicos- los que parecen haber recibido mayor información de los clubes $(73 \%, 69 \%$ y 63\%, respectivamente), algo que puede ser natural, porque los deportistas federados suelen mantener una relación directa y más intensa con las estructuras de los clubes que los deportistas de alto nivel y olímpicos. Hay que añadir, además, que el funcionamiento de los clubes es más voluntarista que profesional - propio de las federaciones-, por lo que es normal que estos siguieran esforzándose en informar a sus asociados durante el confinamiento de forma mucho más clara que las propias federaciones deportivas. 


\begin{tabular}{|c|c|c|}
\hline \multicolumn{3}{|c|}{ Variables } \\
\hline Deportistas / Deportes & $\begin{array}{c}\text { Información Federación } \\
\text { (POCO; NADA) }\end{array}$ & $\begin{array}{c}\text { Información de Club } \\
\text { (MUCHO; BASTANTE) }\end{array}$ \\
\hline Federados & $63 \%$ & $73 \%$ \\
\hline Alto Nivel & $55 \%$ & $69 \%$ \\
\hline Olímpicos & $53 \%$ & $73 \%$ \\
\hline Natación & $64 \%$ & $72 \%$ \\
\hline Remo & $53 \%$ & $63 \%$ \\
\hline
\end{tabular}

Tabla 8. ¿Tu Federación te informaba sobre la evolución de la situación en tu deporte? / ¿Tu club o equipo te informaba sobre la situación en tu deporte? Fuente: Elaboración propia.

En el marco de esas condiciones vividas por los deportistas, se consultó a los participantes en la encuesta acerca de su motivación y el modo de organización de los entrenamientos, en comparación con los momentos previos al comienzo del confinamiento. Comenzando por la primera de esas cuestiones, al preguntarles a los deportistas si fue difícil mantenerse motivado para entrenar, llama la atención que más de la mitad de los encuestados (54\%) respondan que ha sido "nada" o solo "un poco" difícil mantenerse motivado para entrenar en esas condiciones de aislamiento. Si se compara esta actitud según el sexo de las personas encuestadas, es algo mayor esta respuesta entre mujeres que entre hombres (56\% y 52\%, respectivamente). Y, si se analiza teniendo en consideración las categorías de deportistas contempladas en este trabajo, es mayor entre los deportistas de alto nivel que entre los deportistas federados, pero también con respecto a los deportistas olímpicos (57\%, 54\% y 51\%, respectivamente, ver tabla 9). En el caso de los deportistas olímpicos, su respuesta puede estar influida por el modo como haya afectado el confinamiento y la crisis epidemiológica a sus planes para los Juegos Olímpicos de Tokio 2020. En los resultados no se observan diferencias sobre este asunto según el deporte que practiquen, ya sea natación o remo. 


\begin{tabular}{|l|c|}
\hline \multicolumn{2}{|c|}{ Variables } \\
\hline Nivel Deportivo & Sexo \\
\hline Federados: $54 \%$ & \\
Alto Nivel: $57 \%$ & Hombres: $52 \%$ \\
Olímpicos: $51 \%$ & Mujeres: $56 \%$ \\
\hline
\end{tabular}

Tabla 9. ¿Ha sido difícil mantenerse motivado/a para entrenar? Respuesta: MUCHO; BASTANTE. Fuente: Elaboración propia.

Teniendo en cuenta el coste de oportunidad personal y profesional que implica tener una carrera deportiva, la reacción de los deportistas desde el punto de vista de la motivación pone en evidencia la fortaleza del carácter y la voluntad de estos en sus objetivos deportivos. Igualmente muestra el compromiso en sus responsabilidades para con la representación institucional del deporte, incluso en las condiciones más duras y difíciles, como es estar aislado en el hogar siendo deportista de alto nivel u olímpico, y manteniendo la disciplina deportiva desde 6 y hasta más de 16 horas semanales, sin contar con materiales ni equipamientos ni espacios aptos para los ejercicios de entrenamiento.

En virtud de esa apreciación, no extraña que, cuando se les pregunta a los participantes si se han podido organizar para entrenar durante el confinamiento, una amplia mayoría responda "bastante" o "mucho", tanto entre deportistas federados (76\%), como entre deportistas de alto nivel (78\%) y, más aún, entre deportistas olímpicos (84\%). Y algo más también entre mujeres (78\%) que entre hombres $(74 \%)$ y, de nuevo, mucho más entre los deportistas de remo (84\%) que entre los de natación (74\%) (ver tabla 10).

\begin{tabular}{|c|c|c|}
\hline \multicolumn{3}{|c|}{ Variables } \\
\hline Nivel Deportivo & Sexo & Disciplina Deportiva \\
\hline Federados: $74 \%$ & Hombres: $74 \%$ & Natación: 74\% \\
Alto Nivel: $78 \%$ & Mujeres: $78 \%$ & Remo: $84 \%$ \\
Olímpicos: $84 \%$ & & \\
\hline
\end{tabular}

Tabla 10. ¿Te has podido organizar para entrenar durante el confinamiento? Respuesta: MUCHO; BASTANTE. Fuente: Elaboración propia. 
En esta línea, al preguntarles si han perdido estado de forma en comparación con el momento en que comenzó el confinamiento, más de la mitad de los deportistas federados, de alto nivel y olímpicos creen que lo han perdido solo "un poco" o "nada" (54\%, 52\% y 50\%, respectivamente) (ver tabla 11). Aunque las similitudes en esta respuesta entre hombres y mujeres no provocan diferencias, la distinción es muy significativa entre deportistas de remo y deportistas de natación (16 puntos de diferencia, un 67\% los deportistas de remo y un $51 \%$ los deportistas de natación).

\begin{tabular}{|c|c|}
\hline \multicolumn{2}{|c|}{ Variables } \\
\hline Nivel Deportivo & Deportistas por disciplinas \\
\hline Federados: $54 \%$ & \\
Alto Nivel: $52 \%$ & Natación: $51 \%$ \\
Olímpicos: $50 \%$ & Remo: $67 \%$ \\
\hline
\end{tabular}

Tabla 11. ¿Has perdido estado de forma en comparación a cuando empezó el confinamiento? Respuesta: UN POCO; NADA. Fuente: Elaboración propia.

Para finalizar, la encuesta planteó dos preguntas de opinión dirigidas directamente a deportistas olímpicos. Con ellas se trataba de indagar acerca de su posición sobre la suspensión de los Juegos Olímpicos Tokio 2020 y, al mismo tiempo, conocer si se encontraban afectados por dicha suspensión. Llama la atención la casi absoluta unanimidad al considerar adecuada la suspensión de los Juegos Olímpicos de Tokio. Un 98\% lo piensa así, sin apenas diferencia entre deportistas olímpicos hombres y mujeres, y practicantes de natación y de remo. Y esta posición de unanimidad tiene lugar a pesar de que el 34\% de los deportistas olímpicos participantes en la encuesta manifiestan haberse visto afectados por la suspensión de los Juegos Olímpicos de 2020. Puede que algunos estuvieran en condiciones óptimas para participar en estos Juegos, puede que otros se encontrasen en una edad y categoría en la que pudieran verse excluidos al aplazarse, o puede que tuvieran que participar todavía en pruebas decisorias para asistir a Tokio. De entre estos deportistas, son más mujeres que hombres los que expresan haberse visto afectados "mucho" o "bastante" por la suspensión de estos Juegos, un 35\% frente a un $28 \%$. Algo en lo que tiene que ver, probablemente, la mayor dificultad de la mujer en la carrera deportiva profesional y el mayor número de mujeres que de hombres que forman parte de grupo de deportistas olímpicos. Por lo demás, no cambia la distribución general 
por el tipo de disciplinas deportivas de estos deportistas olímpicos de las analizadas en este trabajo (natación o remo).

En contra de lo que dicta el sentido común, pese al enorme coste de oportunidad que acarrea a estos deportistas la suspensión de los Juegos Olímpicos de Tokio 2020 —casi tanto como el de sus propias carreras deportivas-, la situación causada por la crisis epidemiológica del coronavirus provocó muchas reacciones espontáneas por parte de deportistas olímpicos de todo el mundo, que se organizaron y emitieron declaraciones públicas denunciando la presión que estaban recibiendo por parte de federaciones internacionales y patrocinadores para competir a toda costa en los eventos deportivos previstos (Donnelly, 2020).

\section{CONCLUSIONES}

Pese a la existencia de una tradición académica preocupada por el estudio social del deporte, bajo ningún supuesto conocido en nuestra historia reciente, estas disciplinas se habían expuesto al estudio sistemático de hábitos, comportamientos y actitudes deportivas en contextos de aislamiento social. En ese sentido, puede decirse que el confinamiento vivido por estos deportistas a causa de la crisis epidemiológica del coronavirus ha sido un experimento social en toda regla también para la psicología del deporte, del mismo modo que para otras disciplinas de las ciencias sociales que tienen al deporte por objeto, como la antropología y la sociología.

Merece reconocerse el acierto de la decisión estratégica de realizar la recogida de información por parte del equipo coordinado por Jaenes et al. (2020), dado que el confinamiento vivido en la primavera de 2020 no tiene parangón como experiencia social, también para el mundo del deporte, especialmente para el deporte de alto nivel y olímpico. Y ello es así incluso con las limitaciones, deficiencias y posibles errores derivados de administrar una encuesta con las herramientas utilizadas en este estudio para la recogida de datos y que se han enumerado con detalle en este artículo. No obstante lo cual, se ha tratado de minimizar su impacto en las decisiones técnicas adoptadas para la explotación de los datos. Pero también es cierto que no ha de ignorarse la imposibilidad de recoger información social desde la imprevisión de una crisis de tal calado, más aún al no disponer de fuente de financiación de convocatoria o proyecto alguno, y por el establecimiento de un estado de alarma que impidió cualquier tipo de actividad económica y profesional no esencial durante dicho confinamiento.

Los datos explotados en este trabajo no se corresponden con todos los datos obtenidos de la administración de la encuesta. De las 43 preguntas que componían el cuestionario, en este trabajo no se han utilizado más que 25 de ellas (incluyendo las sociodemográficas), ciñéndonos exclusivamente a analizar las condiciones de hábitat, familiares y ocio vividas durante el confinamiento, los hábitos y comportamientos de entrenamiento, las 
actitudes relacionadas con la motivación y organización de sus entrenamientos, así como la disponibilidad de materiales, el seguimiento de entrenadores y la información recibida por federaciones y clubes deportivos y, en última instancia, su posición y el modo como les ha afectado la suspensión de los Juegos Olímpicos de Tokio 2020. Sin embargo, quedan por analizar los resultados de las preguntas sobre su adaptación psicológica, cognitiva y emocional, que han sido recogidos en algunos trabajos publicados (Jaenes et al., 2020; Jaenes et al, 2020a) y otros en espera de publicación.

Los resultados de este trabajo permiten confirmar aquello que a menudo bulle en el imaginario colectivo: que los deportistas (sean federados, de alto nivel u olímpicos) están hechos de una madera especial. Analizados los datos, queda patente el compromiso de nuestros deportistas con sus metas deportivas y su responsabilidad al respetar las indicaciones de confinamiento. Mantenerse entrenando sin la certeza de un calendario de competiciones no es tarea fácil.

Todos los deportistas (federados, de alto nivel y olímpicos) entrenaron durante el confinamiento. Todos, con independencia del tamaño de sus viviendas y contaran o no con terraza o jardín, y disfrutaran o no de apoyo y seguimiento de entrenadores o técnicos. Unos entrenaron más horas -incluso más de 16 horas semanales-, otros menos - entre 5 y 7 horas a la semana-, pero todos se esforzaron por entrenar pese a la falta de materiales - del que carecían entre 6 y 7 de cada 10 deportistas-, o la ausencia de información de sus federaciones o de sus clubes. La mitad, como hemos subrayado, no recibió ningún tipo de información durante ese período y siguió entrenando de forma autónoma. Hablamos, además, de unos deportistas con una peculiaridad, puesto que necesitan instalaciones acuáticas para sus ejercicios, lo que hace más valiosa su dedicación, más aún cuando son personas que compiten en muchos casos al más alto nivel.

Pese a vivir un contexto de tal presión — no olvidemos que, para mayor preocupación, casi 4 de cada 10 deportistas han visto como alguno de sus familiares perdía su trabajo-, la mitad de esos deportistas afirma que ha tenido "ninguna" o solo "un poco" de dificultad para mantener la motivación por el entrenamiento y casi 8 de cada 10 expresan haberse organizado con "mucha" o "bastante" facilidad. La verdad es que es difícil dejar de advertir que, cuando en una sociedad en la que a diario se comprueba que individuos con todos los recursos a su alcance carecen de motivación y falta de orden para cumplir con sus responsabilidades, verificar que existen situaciones inversas como la de estos deportistas representa un estímulo digno de ejemplo para el conjunto de la sociedad. El colofón a esta observación es la posición responsable tanto para cumplir de forma unánime las indicaciones de aislamiento en casa como incluso posicionarse a favor de la suspensión de los Juegos Olímpicos de Tokio 2020, aun a costa del perjuicio personal y profesional de quienes aspiraban o se encontraban llamados a participar en tales Juegos. 
Con todo, el presente trabajo ofrece la oportunidad comedida de conocer un poco mejor a nuestros deportistas en la peor de las circunstancias posibles y en coyunturas vividas ni conocidas hasta ahora. Aun con los márgenes de prudencia que hemos de tomarnos con la herramienta utilizada para la obtención de los datos, creemos que las medidas adoptadas para la selección definitiva de casos con vistas a la indagación científica, garantiza la suficiente representatividad de los mismos para analizar el objeto del trabajo. Y también brinda una buena oportunidad al debate sobre el empleo de este tipo de herramientas de investigación (las encuestas web) para afrontar futuras investigaciones sociales. Para finalizar, no cabe duda de que el trabajo realizado suministra datos de interés para planificar las estrategias más adecuadas en materia de deporte federado, de alto nivel y olímpico, ante eventuales pandemias que puedan producirse en el futuro. 


\section{REFERENCIAS BIBLIOGRÁFICAS}

Andrade, Emilio et al. (2013) "Versión breve en español del cuestionario POMS para deportistas adultos y población general". Cuadernos de Psicología del Deporte, 22(1): 9510 .

Callegaro, Mario; Katja Lozar Manfreda, y Vasja Vehovar (2015) Web Survey Methodology. London: SAGE.

Ciddi, Pinar, y Elif Yazgan (2020) "Investigation of the continuity of training and mental health of athletes during social isolation in the Covid-19 outbreak". International Journal of Disabilities Sports \& Health Science, 3(2): 111-120.

Clemente-Suárez, Vicente Javier et al. (2020) "Modulators of the personal and professional threat perception of olympic athletes in the actual COVID-19 crisis". Frontiers in Psychology, 11: 1985.

Cruz Feliu, Jaume (1992) "El asesoramiento y la intervención psicológica en deportistas olímpicos”. Revista de Psicología del Deporte, 1(2): 42-46.

Di Fronso, Selenia et al. (2020) "The effects of COVID-19 pandemic on perceived stress and psychobiosocial states in Italian athletes". International Journal of Sport and Exercise Psychology, Junio, 1-13.

Díaz de Rada, Vidal (2012) "Ventajas e inconvenientes de la encuesta por Internet". Papers. Revista de Sociología, 97(1): 193-223.

Díaz de Rada, Vidal (2011) "Encuestas con encuestador y autoadministradas por internet. ¿Proporcionan resultados comparables?”. Revista Española de Investigaciones Sociológicas, 136: 49-90.

Díaz de Rada, Vidal; Juan Antonio Domínguez, y Sara Pasadas (2019) Internet como modo de administración de encuestas. Madrid: Centro de Investigaciones Sociológicas (CIS).

Díaz de Rada, Vidal, y Juan Antonio Domínguez (2017) "Comparación de métodos de campo en la encuesta”. Revista Española de Investigaciones Sociológicas, 158: 137-148.

Donnelly, Peter (2020) "We are the games: The COVID-19 pandemic and athletes' voices”. Sociología del Deporte, 1(1): 35-40.

García Ferrando, Manuel; Arturo Oliver, y Juan Ramón Martínez (1996) Los deportistas olímpicos españoles: un perfil sociológico. Madrid: Consejo Superior de Deportes.

Guilherme, Flávio Ricardo et al. (2020) "Perceptive changes in endurance athletes during social isolation due to Covid-19". Revista Brasileira de Medicina do Esporte, 26(6): 473477. 
Jaenes, José Carlos et al. (2020) “El entrenamiento, un moderador de reacciones emocionales en el confinamiento por COVID-19 en deportistas de alto rendimiento?". Revista Andaluza de Medicina del Deporte, 13(3): 120-121.

Jaenes, José Carlos et al. (2020a) "El impacto del confinamiento en nadadores de nivel nacional e internacional". Revista Comunicaciones Técnicas de la Real Federación Española de Natación, 2(2): 41-44.

Moscoso, David, y Eduardo Moyano (2010) Condiciones de vida y expectativas de retorno de los andaluces en el exterior. Sevilla, Consejería de Justicia y Gobernación, Junta de Andalucía.

Ozen, Gulsen; Han Koc, y Cihat Aksoy (2020) "Health anxiety status of elite athletes in COVID-19 social isolation period". Bratislavske Lekarske Listy, 121(12): 888-893.

Ramos e Côrte, Ana Carolina et al. (2020) "Covid-19 and its effect on Olympic sport: the importance of studying social isolation and the harm it causes, in order to minimize it". Revista Brasileira de Medicina do Esporte, 26(5).

Reardon, Claudia et al. (2020) "Mental health management of elite athletes during COVID-19: a narrative review and recommendations". British Journal of Sports Medicine, 0: $1-10$.

Schinke, Robert et al. (2020) "Sport psychology services to high performance athletes during COVID-19". International Journal of Sport and Exercise Psychology, 18(3): 269272.

Serpa, Sidónio, y Tiago Castro (2006) "Psicología de los juegos olímpicos: la percepción de los entrenadores”. Revista de Psicología del Deporte, 15(2): 183-199.

Vilanova, Anna, y Marisol Casado (2000) "El deporte de alto nivel en España. Una comparación a nivel internacional". En Nuria Puig y Andreu Camps (Coord), Diálogos sobre el deporte en España (1980-2020). Barcelona: Editorial INDE. 